\title{
Continuity of the Optimal Stopping Boundary for Two- Dimensional Diffusions
}

DOI:

10.1214/18-AAP1426

\section{Document Version}

Accepted author manuscript

Link to publication record in Manchester Research Explorer

\section{Citation for published version (APA):}

Peskir, G. (2018). Continuity of the Optimal Stopping Boundary for Two-Dimensional Diffusions. Annals of Applied Probability, 29(1), 505-530. https://doi.org/10.1214/18-AAP1426

\section{Published in:}

Annals of Applied Probability

\section{Citing this paper}

Please note that where the full-text provided on Manchester Research Explorer is the Author Accepted Manuscript or Proof version this may differ from the final Published version. If citing, it is advised that you check and use the publisher's definitive version.

\section{General rights}

Copyright and moral rights for the publications made accessible in the Research Explorer are retained by the authors and/or other copyright owners and it is a condition of accessing publications that users recognise and abide by the legal requirements associated with these rights.

\section{Takedown policy}

If you believe that this document breaches copyright please refer to the University of Manchester's Takedown Procedures [http://man.ac.uk/04Y6Bo] or contact uml.scholarlycommunications@manchester.ac.uk providing relevant details, so we can investigate your claim.

\section{OPEN ACCESS}




\title{
Continuity of the Optimal Stopping Boundary for Two-Dimensional Diffusions
}

\author{
Goran Peskir
}

\begin{abstract}
We first show that a smooth fit between the value function and the gain function at the optimal stopping boundary for a two-dimensional diffusion process implies the absence of boundary's discontinuities of the first kind (the right-hand and left-hand limits exist but differ). We then show that the smooth fit itself is satisfied over the flat portion of the optimal stopping boundary arising from any of its hypothesised jumps. Combining the two facts we obtain that the optimal stopping boundary is continuous whenever it has no discontinuities of the second kind. The derived fact holds both in the parabolic and elliptic case under the sole hypothesis of Hölder continuous coefficients, thus improving upon all known results in the parabolic case, and establishing the fact for the first time in the elliptic case. The method of proof relies upon regularity results for the second order parabolic/elliptic PDEs and makes use of the local time-space calculus techniques.
\end{abstract}

\section{Introduction}

A challenging question in problems of optimal stopping is to establish regularity of the optimal stopping boundary separating the continuation set from the stopping set in the problem. By regularity we mean continuity, Lipschitz/Hölder continuity, differentiability, and/or a higher degree of smoothness. The problem has a long history and we refer to [14] and [7] for further historical facts/details and references.

In this paper we consider a general optimal stopping problem for a two-dimensional diffusion process and focus on the question of establishing continuity of the optimal stopping boundary. This is motivated by the fact that in recent years several specific problems of this kind have appeared in the literature (cf. [6], [8], [10], [12], [16], [17]) and further ones are on their way. Often these papers are motivated by real-world applications where dimension two (or higher) plays a crucial role (cf. [16], [17]). This necessitates in establishing general results implying continuity of the optimal stopping boundary that would be applicable in these and similar other problems. Moreover, the known uniqueness arguments [22, Chap. VI-VIII], originally established in [19] and further refined in [11], extend to this fully two-dimensional case as well (cf. [6], [8], [12], [16], [17]) and this enables one to characterise the optimal stopping boundary as the unique solution to a nonlinear integral equation in the class of continuous functions (with the value function being expressed in terms of the optimal stopping boundary itself). This characterisation of the optimal stopping boundary is known to be sufficient for

Mathematics Subject Classification 2010. Primary 60G40, 60J60, 60H30. Secondary 35K20, 35J25, 35R35.

Key words and phrases: Optimal stopping, free boundary, continuity of the optimal stopping boundary, two-dimensional diffusion process, smooth fit, second order parabolic/elliptic PDE, local time-space calculus. 
practical purposes of optimal stopping (using Picard iteration for numerics for instance) and higher degrees of regularity can then be studied subsequently as/if needed. The question of continuity is also of interest in itself due to its deep connections to the interplay between probability (stochastic processes) and analysis (differential equations) yielding the combined results presented at the end of the paper.

Despite the fact that continuity of the optimal stopping boundary has been established in quite a few specific optimal stopping problems studied in the literature, often the arguments used are ad-hoc and a more systematic approach yielding general results of this kind has not been fully developed in the literature. An early paper of this kind, addressing higher degrees of regularity, is due to Friedman [14] (see also [3] and [4, Chapter 8] for related results in higher dimensions). A recent paper of this kind, addressing continuity of the optimal stopping boundary, is due to De Angelis [7]. Both papers deal only with the case when the infinitesimal generator of the process is parabolic while in the elliptic case there are only a few isolated examples (see e.g. [6] and [8] among others).

The purpose of the present paper is to fill in this gap in the literature. In contrast to [7] where the method of proof makes use of the adjoint infinitesimal generator and thus requires a higher degree of smoothness on the coefficients, we develop a new method of proof which relies upon regularity results for the second order parabolic/elliptic PDEs and requires only Hölder continuous coefficients. Moreover, unlike [7] we connect the continuity problem to the principle of smooth fit (stating that the first-order partial derivatives of the value function and the gain function coincide at the optimal stopping boundary). The reason for invoking this connection is twofold. Firstly, the smooth fit is known to be a key variational principle with the power of determining the optimal stopping boundary uniquely among all admissible candidates. In the light of this fact we show that the smooth fit itself implies the continuity of the optimal stopping boundary whenever it has no discontinuities of the second kind. Secondly, regularity of the points at the optimal stopping boundary (for the stopping set) is known to imply the smooth fit under further regularity conditions on the process (cf. [9]). Given that any hypothesised jumps of the first kind correspond to flat portions of the optimal stopping boundary which therefore ought to be regular for the stopping set, we show that the smooth fit itself is satisfied in this setting. To avoid imposing the additional regularity conditions on the process mentioned above, we derive this fact differently by making use of the (semimartingale) local time-space calculus techniques instead. Combining the two facts we obtain that the optimal stopping boundary is continuous whenever it has no discontinuities of the second kind. The derived fact holds both in the parabolic and elliptic case under the sole hypothesis of Hölder continuous coefficients, thus improving upon all known results in the parabolic case, and establishing the fact for the first time in the elliptic case.

More specifically, the paper is organised as follows. In Section 2 we introduce the setting of the problem and explain its background in terms of general hypotheses. These hypotheses are further refined in the following sections. In Section 3 we show that a horizontal smooth fit between the value function and the gain function at the optimal stopping boundary implies the absence of boundary's discontinuities of the first kind (the right-hand and left-hand limits exist but differ). The method of proof in the parabolic case relies upon regularity results for the second order parabolic PDEs that make it possible to calculate the vertical partial derivatives of the value function along the flat portion of the optimal stopping boundary arising from any of its hypothesised jumps. This argument breaks down for elliptic equations due to the existence 
of the second horizontal derivative in that case. The method of proof in the elliptic case relies upon a trick which makes it possible to apply Hopf's boundary point lemma for elliptic equations in the perpendicular direction to the flat portion of the optimal stopping boundary arising from any of its hypothesised jumps. It is interesting to note that this argument in turn breaks down for parabolic equations due to the absence of an interior sphere condition in that direction. In the parabolic case we also derive a general sufficient condition for the absence of boundary's discontinuities of the first kind that goes beyond smooth fit. In Section 4 we show that a horizontal smooth fit between the value function and the gain function is satisfied over the flat portion of the optimal stopping boundary arising from any of its hypothesised jumps. In essence this can be seen as a consequence of the fact that points at the flat boundary ought to be regular for the stopping set. In this section we develop a different line of argument in the elliptic case and show that this can be also derived under weaker hypotheses from the fact that the first component of the process develops a nontrivial (semimartingale) local time having a square-root growth at zero after starting at the flat boundary. This argument relies upon the change-of-variable formula with local times on surfaces [21] and has been used in an earlier proof of [19, p. 178] when the infinitesimal generator is parabolic. Given that this formula holds for 'semimartingale' surfaces (whose points are not necessarily regular for the stopping set) the local time argument itself highlights an interesting connection between the regularity of boundary points and the existence of a nontrivial local time (cf. [2, p. 216]). The paper concludes with corollaries obtained by merging the results of Sections 3 and 4 .

\section{Optimal stopping problem}

In this section we introduce the setting of the problem and explain its background in terms of general hypotheses. These hypotheses will be further refined in the next section.

1. We consider the optimal stopping problem

$$
V(x, y)=\sup _{\tau} \mathrm{E}_{x, y}\left[e^{-\Lambda_{\tau}} G\left(X_{\tau}, Y_{\tau}\right)+\int_{0}^{\tau} e^{-\Lambda_{t}} H\left(X_{t}, Y_{t}\right) d t\right]
$$

where $(X, Y)$ is a two-dimensional diffusion process solving

$$
\begin{aligned}
& d X_{t}=\mu_{1}\left(X_{t}, Y_{t}\right) d t+\sigma_{11}\left(X_{t}, Y_{t}\right) d B_{t}^{1}+\sigma_{12}\left(X_{t}, Y_{t}\right) d B_{t}^{2} \\
& d Y_{t}=\mu_{2}\left(X_{t}, Y_{t}\right) d t+\sigma_{21}\left(X_{t}, Y_{t}\right) d B_{t}^{1}+\sigma_{22}\left(X_{t}, Y_{t}\right) d B_{t}^{2}
\end{aligned}
$$

with $\left(X_{0}, Y_{0}\right)=(x, y)$ in $\mathbb{R}^{2}$ under the probability measure $\mathrm{P}_{x, y}$ where $B=\left(B^{1}, B^{2}\right)$ is a standard two-dimensional Brownian motion. Setting $Z=\left(Z_{1}, Z_{2}\right):=(X, Y)$ we see that the system $(2.2)+(2.3)$ can be written in a compact matrix form as follows

$$
d Z_{t}=\mu\left(Z_{t}\right) d t+\sigma\left(Z_{t}\right) d B_{t}
$$

where $\mu=\left(\mu_{1}, \mu_{2}\right)$ denotes the drift vector and $\sigma=\left(\sigma_{11} \sigma_{12} ; \sigma_{21} \sigma_{22}\right)$ denotes the diffusion coefficient matrix (written as a sequence of two of its rows). We will assume that $Z$ is a unique weak solution to (2.4) so that $Z$ is a strong Markov process with respect to the underlying filtration that makes $B$ a standard two-dimensional Brownian motion. The assumption that $Z$ solves (2.4) is not crucial and on more careful inspection one can see that the results below 
hold for general continuous (strong) Markov processes with drift $\mu$ and diffusion coefficient $\sigma$ given that the other hypotheses ( on $\mu$ and $\sigma$ ) introduced below are satisfied.

2. The supremum in (2.1) is taken over all stopping times $\tau$ of $Z=(X, Y)$ (i.e. stopping times with respect to the natural filtration of $Z$ ), or equivalently, over all stopping times $\tau$ with respect to a filtration that makes $Z$ a strong Markov process (as in the weak solution description of $Z$ above). All stopping times considered throughout are assumed to be finite valued, and we impose no upper bound on $\tau$ in the optimal stopping problem (2.1), i.e. its horizon is infinite unless $\mu_{1} \equiv 1$ and $\sigma_{11}=\sigma_{12} \equiv 0$ (when $Z$ is a time-space diffusion process) in which case the horizon can also be finite. The functional $\Lambda$ in (2.1) is defined by

$$
\Lambda_{t}=\int_{0}^{t} \lambda\left(X_{s}, Y_{s}\right) d s
$$

where $\lambda$ is a continuous function with values in $[0, \infty)$. The real-valued functions $G$ and $H$ are also assumed to be continuous. Under these hypotheses it is known (cf. [22, p. 46] and [24, p. 127]) that the first entry time of $Z$ into the (stopping) set $D$ where $V$ equals $G$ is optimal in (2.1) provided that $G(Z)$ and $H(Z)$ satisfy mild integrability conditions. This is true for example if $\lambda>0$ and both $G$ and $H$ are bounded but this sufficient condition can be considerably strengthened (see [22] and [24] for details). The (continuation) set where $V$ is strictly larger than $G$ will be denoted by $C$. The (optimal stopping) boundary between the sets $C$ and $D$ will be denoted by $\partial C$. Moreover, we will assume that $G$ satisfies

$$
\mathrm{E}_{x, y}\left[e^{-\Lambda_{\tau}} G\left(X_{\tau}, Y_{\tau}\right)\right]=G(x, y)+\mathrm{E}_{x, y}\left[\int_{0}^{\tau} e^{-\Lambda_{t}}\left(\mathbb{L}_{Z} G-\lambda G\right)\left(X_{t}, Y_{t}\right) d t+A_{\tau}\right]
$$

for all (bounded) stopping times $\tau$ of $Z$ and all $(x, y)$ in the state space of $Z$. The symbol $\mathbb{L}_{Z}$ in (2.6) denotes the infinitesimal generator of $Z$ given by

$$
\mathbb{L}_{Z}=\alpha \partial_{x x}+2 \beta \partial_{x y}+\gamma \partial_{y y}+\mu_{1} \partial_{x}+\mu_{2} \partial_{y}
$$

where by $(2.2)+(2.3)$ one can verify using Itô's calculus that

$$
\alpha=\frac{1}{2}\left(\sigma_{11}^{2}+\sigma_{12}^{2}\right), \beta=\frac{1}{2}\left(\sigma_{11} \sigma_{21}+\sigma_{12} \sigma_{22}\right), \gamma=\frac{1}{2}\left(\sigma_{21}^{2}+\sigma_{22}^{2}\right)
$$

in the state space of $Z$. The process $A$ in (2.6) is assumed to be continuous with $A_{0}=0$ (under each $\mathrm{P}_{x, y}$ ), adapted to the natural filtration of $Z$, and satisfying that for every $z \in \partial C$ and $\varepsilon>0$ there exist $z^{\prime} \in b(z, \varepsilon) \cap \partial C$ and $\varepsilon^{\prime}>0$ (small enough) such that $A$ is constant (zero) while $Z$ is in $b\left(z^{\prime}, \varepsilon^{\prime}\right.$ ) (throughout the paper $b(c, r)$ denotes the open ball in Euclidean topology with centre at $c$ and radius $r$ ). Itô's formula and the optional sampling theorem imply that (2.6) holds (with $A \equiv 0$ ) when $G$ is $C^{2}$. Using the change-of-variable formula with local time on curves/surfaces (see [20] and [21]) one sees that (2.6) holds with A satisfying the specified conditions (as the integral with respect to a local time) even when $G_{x}, G_{y}, G_{x x}$, $G_{y y}$ have discontinuities (of the first kind) over finitely many continuous curves which intersect $\partial C$ in $b(z, r)$ at finitely many points for $z \in \partial C$ and $r>0$ sufficiently small.

3. Due to (2.6) we know that the Bolza formulated problem (2.1) can be reformulated in the Lagrange form (see [22, p. 141] for the terminology). This is obtained by setting

$$
\hat{H}:=\mathbb{L}_{Z} G-\lambda G+H
$$


and considering the optimal stopping problem

$$
\hat{V}(x, y)=\sup _{\tau} \mathrm{E}_{x, y}\left[\int_{0}^{\tau} e^{-\Lambda_{t}} \hat{H}\left(X_{t}, Y_{t}\right) d t+A_{\tau}\right]
$$

where the supremum is taken over all stopping times $\tau$ of $Z=(X, Y)$ and $(x, y)$ belongs to the state space of $Z$. From (2.1) and (2.6) we then see that

$$
\hat{V}(x, y)=V(x, y)-G(x, y)
$$

for all $(x, y)$ in the state space of $Z$ and a stopping time is optimal in (2.1) if and only if it is optimal in (2.10). We thus focus on the optimal stopping problem (2.10) in the sequel.

4. The functions $\mu_{i}$ and $\sigma_{i j}$ in (2.2) and (2.3) are assumed to be continuous and $\sigma_{i j}$ is assumed to be non-negative for $i, j=1,2$. Although some $\sigma_{i j}$ can be zero we always assume that $\operatorname{tr}(\sigma)=\sigma_{11}+\sigma_{12}+\sigma_{21}+\sigma_{22}>0$ (i.e. at least one of the Brownian motions $B^{1}$ and $B^{2}$ will be driving at least one of the equations (2.2) and (2.3)). Note that this includes the case when $X$ is of bounded variation (i.e. $\sigma_{11}=\sigma_{12} \equiv 0$ ) as well as the case of a time-space diffusion $\left(t, Y_{t}\right)_{t \geq 0}$ (when $\mu_{1} \equiv 1$ as well). Moreover, from $(2.7)+(2.8)$ we see that

$$
\beta^{2}-\alpha \gamma=-\frac{1}{4}(\operatorname{det}(\sigma))^{2} \leq 0
$$

which shows that the partial differential operator (2.7) is of parabolic type when $\operatorname{det}(\sigma)=0$ and elliptic type when $\operatorname{det}(\sigma) \neq 0$. In the former (parabolic) case there is no restriction in assuming that $\alpha \equiv 0$ and $\beta \equiv 0$. Indeed, this can be achieved by passage to the canonical equation. For this, consider the characteristic curves equation $d y / d x=\beta / \alpha$ and (upon assuming that $y \mapsto(\beta / \alpha)(x, y)$ is locally Lipschitz) write down its general solution in the form $S(x, y)=c$ where $c$ is an arbitrary constant. Changing then variables as $x^{\prime}=S(x, y)$ and $y^{\prime}=y$ it can be verified using (2.8) above that the diffusion coefficient matrix of the resulting process $Z^{\prime}=\left(X^{\prime}, Y^{\prime}\right)$ yields $\alpha^{\prime} \equiv 0$ and $\beta^{\prime} \equiv 0$ as claimed. In the latter (elliptic) case there is no restriction in assuming that $\alpha \equiv \gamma$ and $\beta \equiv 0$. Indeed, this can be similarly achieved by passage to the canonical equation. For this, consider the characteristic curves equation $d y / d x=\left(\beta \pm \sqrt{\beta^{2}-\alpha \gamma}\right) / \alpha$ and (upon assuming that $y \mapsto\left(\left(\beta \pm \sqrt{\beta^{2}-\alpha \gamma}\right) / \alpha\right)(x, y)$ is locally Lipschitz) write down its general solution as $S_{ \pm}(x, y)=c_{ \pm}$where $c_{ \pm}$is an arbitrary constant. Changing then variables as $x^{\prime}=S_{+}(x, y)+S_{-}(x, y)$ and $y^{\prime}=\sqrt{-1}\left(S_{+}(x, y)-S_{-}(x, y)\right)$ it can be verified using (2.8) above that the diffusion coefficient matrix of the resulting process $Z^{\prime}=\left(X^{\prime}, Y^{\prime}\right)$ yields $\alpha^{\prime} \equiv \gamma^{\prime}$ and $\beta^{\prime} \equiv 0$ as claimed. We will see in the proof below however that the passage to the canonical equation is not needed in the elliptic case.

5. The optimal stopping boundary $\partial C$ in (2.1) can generally take various shapes and forms. In this paper we will focus on the case when $\partial C$ can be locally represented as the graph of a function $\mathrm{b}$ in the state space of $Z=(X, Y)$. This fact is known to be satisfied in specific problems of optimal stopping and leads to characterisations of the optimal stpping boundaries as unique solutions to nonlinear integral equations (cf. Section 1). Without loss of generality we will assume that $\mathrm{b}$ is a function of the first coordinate $x$ belonging to a (sufficiently small) open interval $I$. The problem then arises to determine whether the function $\mathrm{b}$ is continuous at a given point $x_{0}$ in $I$. The first step in this direction may consist of showing that the function $\mathrm{b}$ has no discontinuities of the second kind. In specific optimal stopping problems 


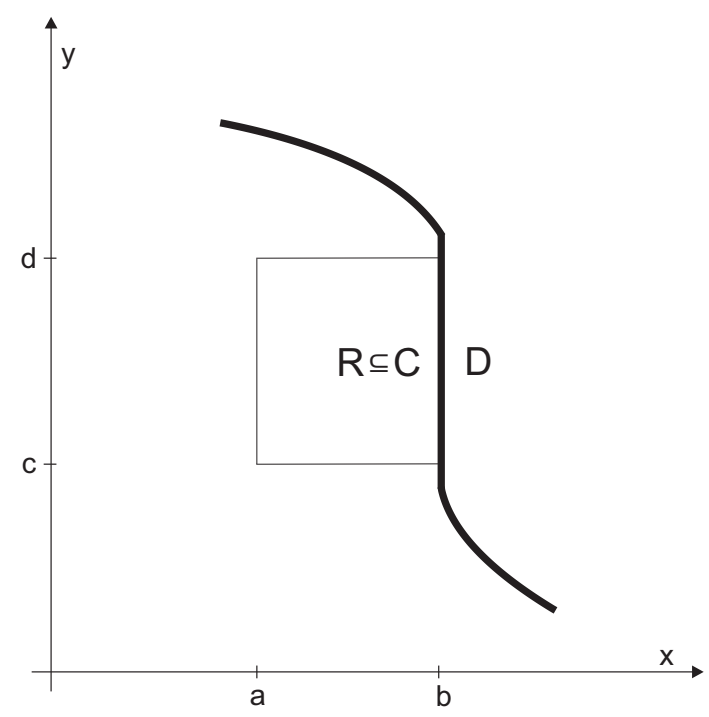

Figure 1 (A boundary discontinuity of the first kind). A rectangle $R:=[a, b] \times[c, d]$ in the state space of $(X, Y)$ obtained by choosing $a<b$ sufficiently close to $b$ and $[c, d]$ sufficiently small with $c<d$ so that $[a, b) \times[c, d] \subseteq C$ with $\{b\} \times[c, d] \subseteq D$.

this fact is frequently established by showing that the function $\mathrm{b}$ is (locally) monotone or (locally) of bounded variation. The question of finding general conditions under which $\mathrm{b}$ has no discontinuities of the second kind at $x_{0}$ will not be considered in the present paper (see [14] for general results of this kind). The second step in deriving the continuity of $b$ then reduces to showing that $\mathrm{b}$ has no discontinuities of the first kind at $x_{0}$. This amounts to disproving the existence of vertical segments in the state space of $Z$ which form part of the optimal stopping boundary $\partial C$. Indeed, if $\mathrm{b}$ has a discontinuity of the first kind at $x_{0}$, it means that both $\mathrm{b}\left(x_{0}-\right)$ and $\mathrm{b}\left(x_{0}+\right)$ exist but $\mathrm{b}\left(x_{0}-\right) \neq \mathrm{b}\left(x_{0}+\right)$. The segment $\left\{x_{0}\right\} \times\left[\mathrm{b}\left(x_{0}-\right) \wedge \mathrm{b}\left(x_{0}+\right), \mathrm{b}\left(x_{0}-\right) \vee \mathrm{b}\left(x_{0}+\right)\right]$ then forms part of the optimal stopping boundary which vertically separates the continuation set $C$ from the stopping set $D$. Without loss of generality (due to the general sign of $\mu_{1}$ in the parabolic case) we will assume that the points on the left from the segment locally belong to $C$ and the points on the right from the segment locally belong to $D$. Renaming $x_{0}$ to $b$ (which now denotes a real number and should not be confused with the entire function $\mathrm{b}$ itself) this yields a rectangle $R:=[a, b] \times[c, d]$ in the state space of $Z$ obtained by choosing $a<b$ sufficiently close to $b$ and $[c, d] \subseteq$ $\left[\mathrm{b}\left(x_{0}-\right) \wedge \mathrm{b}\left(x_{0}+\right), \mathrm{b}\left(x_{0}-\right) \vee \mathrm{b}\left(x_{0}+\right)\right]$ sufficiently small with $c<d$ so that $[a, b) \times[c, d] \subseteq C$ with $\{b\} \times[c, d] \subseteq D$ (see Figure 1 above) and $A$ from (2.10) is constant (zero) while $Z$ is in $R$. Due to a local character of the continuity question under consideration we will see below that for the latter reason there is no restriction in assuming that $A \equiv 0$ when referring to the optimal stopping problem (2.10).

6. The problem of showing that $\mathrm{b}$ has no discontinuities of the first kind therefore reduces to disproving the existence of a rectangle $R$ with the properties specified above. To this end, in the parabolic case at least, we could also assume that $\hat{H}(z) \neq 0$ for at least one $z$ in $\{b\} \times[c, d]$. Since $\hat{H}$ is continuous and the interval $[c, d]$ can be taken arbitrarily small (with 
$c \neq d)$ we see that this assumption is equivalent to assuming that

$$
\hat{H} \neq 0 \text { on }\{b\} \times[c, d]
$$

where we recall that $\{b\} \times[c, d]$ is contained in $\partial C$. The latter assumption cannot generally be improved in the parabolic case. Indeed, the example discussed in [5, Remark 17] shows that the optimal stopping boundary $\mathrm{b}$ can have discontinuities of the first kind when $\hat{H}$ equals zero on $\{b\} \times[c, d]$. Moreover, if $\hat{H}$ is strictly positive at some $z \in\{b\} \times[c, d]$, then the first exit time from a sufficiently small ball with the centre at $z$ would produce a larger value in (2.10) than stopping at once, which contradicts the fact that $z$ belongs to $D$. This shows that we could also assume that $\hat{H}<0$ in (2.13) but this will not be needed in the sequel.

7. In addition to the regularity hypotheses imposed throughout this section, we will also assume that $V$ is continuous (on $R$ ). This fact can normally be established in specific examples of optimal stopping problems without difficulty and the question of specifying general conditions under which the value function $V$ is continuous will not be considered in the present paper. Recalling that $G$ is continuous we see that this is equivalent to assuming that

$$
\hat{V} \in C(R)
$$

where $C(R)$ denotes the family of continuous real-valued functions on $R$.

8. When the standard regularity hypotheses discussed in this section (including any of the sufficient conditions) are satisfied, we will say that the optimal stopping problem (2.1) is well posed. This will be a standing premise for the rest of the paper. The assumptions (2.13) and (2.14) will always be invoked explicitly in statements of the results below when needed.

\section{Smooth fit implies continuity}

Consider the optimal stopping problem (2.1) upon assuming that it is well posed as discussed in the previous section. In this section we show that a horizontal smooth fit between the value function $V$ and the gain function $G$ at the optimal stopping boundary implies the absence of boundary's discontinuities of the first kind.

1. To formulate the results we will first introduce some definitions. For $z_{1}=\left(x_{1}, y_{1}\right)$ and $z_{2}=\left(x_{2}, y_{2}\right)$ in $\mathbb{R}^{2}$ we define the distance function

$$
d_{\sigma}\left(z_{1}, z_{2}\right)=\left\{\begin{array}{lll}
\left(\left|x_{1}-x_{2}\right|+\left|y_{1}-y_{2}\right|^{2}\right)^{1 / 2} & \text { if } & \operatorname{det}(\sigma)=0 \\
\left(\left|x_{1}-x_{2}\right|^{2}+\left|y_{1}-y_{2}\right|^{2}\right)^{1 / 2} & \text { if } & \operatorname{det}(\sigma) \neq 0
\end{array}\right.
$$

where there is no loss of generality in assuming that $\sigma_{11}=\sigma_{12}=0$ when $\operatorname{det}(\sigma)=0$. Let $S$ be a subset of $\mathbb{R}^{2}$ and let $F: S \rightarrow \mathbb{R}$ be a function. We say that $F$ is (uniformly) Hölder continuous on $S$ (with exponent $p>0$ ) if there exists $K>0$ such that

$$
\left|F\left(z_{1}\right)-F\left(z_{2}\right)\right| \leq K\left(d_{\sigma}\left(z_{1}, z_{2}\right)\right)^{p}
$$

for all $z_{1}, z_{2} \in S$. We say that $F$ is locally Hölder continuous on $S$ (with exponent $p>0$ ) if for every $z \in S$ there exist $r>0$ and $K_{r}>0$ such that

$$
\left|F\left(z_{1}\right)-F\left(z_{2}\right)\right| \leq K_{r}\left(d_{\sigma}\left(z_{1}, z_{2}\right)\right)^{p}
$$


for all $z_{1}, z_{2} \in b(z, r) \cap S$. The family of all (uniformly) Hölder continuous functions on $S$ (with exponent $p>0$ ) will be denoted by $C_{p}(S)$.

2. Parabolic case. If $\operatorname{det}(\sigma)=0$ (parabolic equation) then there is no loss of generality in assuming that the infinitesimal generator of $Z=(X, Y)$ solving (2.2)-(2.4) is given by

$$
\mathbb{L}_{Z}=\gamma \partial_{y y}+\mu_{1} \partial_{x}+\mu_{2} \partial_{y}
$$

as discussed in Section 2 (recall that $\gamma>0$ is given by (2.8) above). Recalling further that $R:=[a, b] \times[c, d]$ let $\partial_{p} R$ denote the parabolic boundary of $R$ given by

$$
\partial_{p} R= \begin{cases}([a, b] \times\{c, d\}) \cup(\{b\} \times[c, d]) & \text { if } \mu_{1}>0 \text { on } R \\ (\{a\} \times[c, d]) \cup([a, b] \times\{c, d\}) & \text { if } \mu_{1}<0 \text { on } R .\end{cases}
$$

For $z \in R$ we define its $d_{\sigma}$-distance to the parabolic boundary $\partial_{p} R$ by

$$
d_{\sigma}\left(z, \partial_{p} R\right)=\inf \left\{d_{\sigma}\left(z, z^{\prime}\right) \mid z^{\prime} \in \partial_{p} R\right\} .
$$

In the sequel we set $R^{0}:=\operatorname{int}(R)=(a, b) \times(c, d)$ and $R^{1}:=(a, b] \times(c, d)$. Note that $R^{1}$ includes the vertical flat portion $\{b\} \times(c, d)$ of the optimal stopping boundary $\partial C$. Let $C_{p, q}\left(R^{0}\right)$ denote the family of locally Hölder continuous functions $F$ on $R^{0}$ (with exponent $p>0$ ) satisfying the following two conditions

$$
\begin{aligned}
& \sup _{z \in R^{0}}\left(d_{\sigma}\left(z, \partial_{p} R\right)\right)^{q}|F(z)|<\infty \\
& \sup _{z_{1}, z_{2} \in R^{0}}\left(d_{\sigma}\left(z_{1}, \partial_{p} R\right) \wedge d_{\sigma}\left(z_{2}, \partial_{p} R\right)\right)^{p+q} \frac{\left|F\left(z_{1}\right)-F\left(z_{2}\right)\right|}{\left(d_{\sigma}\left(z_{1}, z_{2}\right)\right)^{p}}<\infty
\end{aligned}
$$

for $q \geq 0$ given and fixed. Note that $F$ from $C_{p, q}\left(R^{0}\right)$ can explode at the parabolic boundary $\partial_{p} R$ when $q>0$. Recalling that $C^{1,2}\left(R^{0}\right)$ denotes the family of continuous real-valued functions on $R^{0}$ such that $F_{x}, F_{y}, F_{y y}$ exist and are continuous on $R^{0}$, we let $C_{p, 2}^{1,2}\left(R^{0}\right)$ denote the family of functions $F$ in $C^{1,2}\left(R^{0}\right)$ satisfying

$$
F \in C_{p, 0}\left(R^{0}\right), F_{x} \in C_{p, 2}\left(R^{0}\right), F_{y} \in C_{p, 1}\left(R^{0}\right), F_{y y} \in C_{p, 2}\left(R^{0}\right)
$$

for $p>0$ given and fixed.

Definition 1. We say that a horizontal smooth fit holds at $(b, e) \in \partial C$ if $[a, b) \times\{e\} \subseteq C$ for some $a<b$ sufficiently close to $b$ and we have

$$
\frac{\partial \hat{V}}{\partial x}(b-, e)=0 .
$$

Note that this condition is equivalent to

$$
\frac{\partial V}{\partial x}(b-, e)=\frac{\partial G}{\partial x}(b-, e) .
$$

Clearly this is a horizontal smooth fit from the left (which we treat) and one can analogously define (and treat) a horizontal smooth fit from the right. 
Definition 2. We say that the optimal stopping boundary $\partial C$ has a discontinuity of the first kind at $b \in \mathbb{R}$ if $[a, b) \times[c, d] \subseteq C$ and $\{b\} \times[c, d] \subseteq D$ for some $a<b$ sufficiently close to $b$ and some sufficiently small $[c, d]$ with $c<d$ (see Figure 1 above).

The main result of this section in the parabolic case can now be stated as follows (see also Remark 4 and Corollary 5 below).

Theorem 3. Consider the optimal stopping problem (2.1) when $\operatorname{det}(\sigma)=0$ upon assuming that it is well posed so that (2.13) and (2.14) are satisfied. Assume moreover that we have

$$
\gamma, \mu_{1}, \mu_{2}, \lambda, \hat{H} \in C_{p}([a, b] \times[c, d])
$$

for some $p>0$ with some $a<b$ and $c<d$ such that $[a, b) \times[c, d] \subseteq C$ and $\{b\} \times[c, d] \subseteq D$. If the horizontal smooth fit holds at $(b, e) \in \partial C$, then the optimal stopping boundary $\partial C$ has no discontinuity of the first kind at $b$.

Proof. 1. Setting $R:=[a, b] \times[c, d]$ and noting that $C_{p}(R) \subseteq C_{p, q}\left(R^{0}\right)$ for all $q \geq 0$ we see from (3.12) that $\hat{H} \in C_{p, 2}\left(R^{0}\right)$. Moreover, since the horizontal sides of $R$ are graphs (constants) of $C^{2}$ functions on $[a, b]$, and $\hat{V}$ is continuous on $R$, by the existence and uniqueness result for parabolic equations based on the a priori interior estimates of Schauder type [13, Theorem 9, p. 69] we can conclude using (3.12) that the initial-boundary value problem

$$
\begin{aligned}
& \gamma \tilde{V}_{y y}+\mu_{1} \tilde{V}_{x}+\mu_{2} \tilde{V}_{y}-\lambda \tilde{V}=-\hat{H} \quad \text { on } R^{0} \\
& \tilde{V}=\hat{V} \quad \text { on } \partial_{p} R
\end{aligned}
$$

has a unique solution $\tilde{V}$ in $C^{1,2}\left(R^{0}\right) \cap C(R)$ that moreover belongs to $C_{p, 2}^{1,2}\left(R^{0}\right)$.

2. Letting $\tau_{1}=\inf \left\{t \geq 0 \mid Z_{t} \in \partial_{p} R\right\}$ denote the first entry time of $Z$ to $\partial_{p} R$, by Itô's formula applied to $e^{-\Lambda} \tilde{V}(Z)$ stopped at $\tau_{1}$ and the optional sampling theorem (recalling also that $A$ from (2.6) stopped at $\tau_{1}$ equals zero), we find using (3.13) and (3.14) that

$$
\begin{aligned}
\tilde{V}(z) & =\mathrm{E}_{z}\left[e^{-\Lambda_{\tau_{1}}} \tilde{V}\left(Z_{\tau_{1}}\right)\right]+\mathrm{E}_{z}\left[\int_{0}^{\tau_{1}} e^{-\Lambda_{t}} \hat{H}\left(Z_{t}\right) d t\right] \\
& =\mathrm{E}_{z}\left[e^{-\Lambda_{\tau_{1}}} \hat{V}\left(Z_{\tau_{1}}\right)\right]+\mathrm{E}_{z}\left[\int_{0}^{\tau_{1}} e^{-\Lambda_{t}} \hat{H}\left(Z_{t}\right) d t\right]=\hat{V}(z)
\end{aligned}
$$

for all $z \in R^{0}$ where in the final equality we use that $\left(e^{-\Lambda_{t \wedge \tau_{1}}} \hat{V}\left(Z_{t \wedge \tau_{1}}\right)+\int_{0}^{t \wedge \tau_{1}} e^{-\Lambda_{s}} \hat{H}\left(Z_{s}\right) d s\right)_{t>0}$ is a (bounded) martingale as is well known from the general optimal stopping theory for strong Markov processes since $\tau_{1} \leq \tau_{D}$ and $\left(\left(\Lambda_{t}, \int_{0}^{t} e^{-\Lambda_{s}} \hat{H}\left(Z_{s}\right) d s, Z_{t}\right)\right)_{t \geq 0}$ is a strong Markov process (see $[22,(2.2 .12) \&(6.0 .6)]$ ). This shows that $\hat{V}=\tilde{V}$ on $R$ and hence $\hat{V}$ belongs to both $C^{1,2}\left(R^{0}\right) \cap C(R)$ and $C_{p, 2}^{1,2}\left(R^{0}\right)$.

3. Moreover, we claim that

$$
\hat{V} \in C^{1,2}\left(R^{1}\right)
$$

where we recall that $R^{1}=(a, b] \times(c, d)$. Indeed, if $\mu_{1}<0$ at one point from $\{b\} \times(c, d)$ at least, this follows from the fact that $\hat{V} \in C_{p, 2}^{1,2}\left(R^{0}\right)$ since $\{b\} \times(c, d)$ does not intersect the 
parabolic boundary $\partial_{p} R$ in this case. On the other hand, if $\mu_{1} \geq 0$ on $\{b\} \times(c, d)$ then since $\mu_{1}$ is continuous it follows that $0 \leq \mu_{1} \leq K / 2$ on $R$ for some $K>0$ sufficiently large. We can then rewrite $(3.13)+(3.14)$ as follows

$$
\begin{aligned}
& \gamma \tilde{V}_{y y}+\tilde{\mu}_{1} \tilde{V}_{x}+\mu_{2} \tilde{V}_{y}-\lambda \tilde{V}=-\tilde{H} \text { on } R^{0} \\
& \tilde{V}=\hat{V} \quad \text { on } \partial_{p} R
\end{aligned}
$$

where we set $\tilde{\mu}_{1}:=\mu_{1}-K<0$ and $\tilde{H}:=\hat{H}+K \hat{V}_{x}$ on $R$. Recalling what we established above we see that $\gamma, \tilde{\mu}_{1}, \mu_{2}, \lambda \in C_{p}(R)$ and $\hat{H} \in C_{p, 2}\left(R^{0}\right)$. Moreover, from (3.16) we see that $\hat{V}_{x}$ belongs to $C_{p, 1}\left(R^{0}\right) \subseteq C_{p, 2}\left(R^{0}\right)$ so that $\tilde{H}:=\hat{H}+K \hat{V}_{x}$ belongs to $C_{p, 2}\left(R^{0}\right)$ as well. This shows that the existence and uniqueness result for parabolic equations based on the a priori interior estimates of Schauder type [13, Theorem 9, p. 69] is applicable to (3.17) $+(3.18)$ implying the existence of a unique solution $\tilde{V}$ in $C^{1,2}\left(R^{0}\right) \cap C(R)$ that moreover belongs to $C_{p, 2}^{1,2}\left(R^{0}\right)$. Since however $\tilde{\mu}_{1}<0$ on $R$ we see that $\{b\} \times(c, d)$ does not intersect the parabolic boundary $\partial_{p} R$ and hence using the same argument as above we can conclude that $\tilde{V} \in C^{1,2}\left(R^{1}\right)$. Noting on the other hand that $\hat{V}$ itself solves $(3.17)+(3.18)$ and belongs to $C^{1,2}\left(R^{0}\right) \cap C(R)$ we see by the uniqueness claim in the result above that $\hat{V}=\tilde{V}$ and hence (3.16) holds in this case too as claimed.

4. Summarising the facts derived above we know that $\hat{V}$ solves

$$
\gamma \hat{V}_{y y}+\mu_{1} \hat{V}_{x}+\mu_{2} \hat{V}_{y}-\lambda \hat{V}=-\hat{H} \quad \text { on } R^{0}
$$

and belongs to $C^{1,2}\left(R^{1}\right) \cap C(R)$ as established in (3.16) above. Since $\hat{V}=0$ on $\{b\} \times(c, d)$ we see that $\hat{V}_{y}=\hat{V}_{y y}=0$ on $\{b\} \times(c, d)$. Hence using the fact that $\hat{V} \in C^{1,2}\left(R^{1}\right)$ and taking any sequence $b_{n} \uparrow b$ as $n \rightarrow \infty$ we see that $\lim _{n \rightarrow \infty} \hat{V}\left(b_{n}, e\right)=\lim _{n \rightarrow \infty} \hat{V}_{y}\left(b_{n}, e\right)=$ $\lim _{n \rightarrow \infty} \hat{V}_{y y}\left(b_{n}, e\right)=0$. If moreover the horizontal smooth fit holds at $(b, e)$ as assumed, then in addition to the preceding limit relations we also have that $\lim _{n \rightarrow \infty} \hat{V}_{x}\left(b_{n}, e\right)=0$. Thus evaluating both sides in (3.19) at $\left(b_{n}, e\right) \in R^{0}$ and letting $n \rightarrow \infty$ we obtain $0=-\hat{H}(b, e)$ which contradicts that fact that $\hat{H}(b, e) \neq 0$ as stated in (2.13) above. Hence the optimal stopping boundary $\partial C$ cannot have a discontinuity of the first kind at $b$ as claimed.

Remark 4. It can be shown using the methods/hypotheses from [9] that when $\mu_{1}>0$ at $(b, e) \in \partial C$ then the horizontal smooth fit (from the left) holds at $(b, e)$ so that the conclusion of Theorem 3 that the optimal stopping boundary $\partial C$ has no discontinuity of the first kind at $b$ is always satisfied in this case (note that this also follows from $(3.20)$ below since $\hat{V}_{x}(b-, e) \leq 0$ and $\hat{H}(b, e)<0$ as explained following (2.13) above). Moreover, we will see in Example 14 below that the horizontal smooth fit can break down at $(b, e) \in \partial C$ when $\mu_{1}<0$ and the optimal stopping boundary $\partial C$ has a discontinuity of the first kind at $b$ in this case.

The following extract from the proof above addresses all signs of $\mu_{1}$ simultaneously with no reference to smooth fit being in place.

Corollary 5. Consider the optimal stopping problem (2.1) when $\operatorname{det}(\sigma)=0$ upon assuming that it is well posed so that (2.14) is satisfied. Assume moreover that (3.12) holds as stated above and that the following condition is satisfied

$$
\mu_{1}(b, e) \hat{V}_{x}(b-, e) \neq-\hat{H}(b, e)
$$


for at least one point $e \in(c, d)$. Then the optimal stopping boundary $\partial C$ has no discontinuity of the first kind at $b$.

Proof. Proceeding as in the proof of Theorem 3 above and evaluating both sides in (3.19) at $\left(b_{n}, e\right)$ for $b_{n} \uparrow b$ as $n \rightarrow \infty$ and $e \in(c, d)$ at which (3.20) holds, we find that $\mu_{1} \hat{V}_{x}=-\hat{H}$ at $(b, e)$. This conclusion, however, contradicts (3.20) itself and completes the proof.

Remark 6. The result of Corollary 5 contains the results of Theorems 3.1-3.3 in [7] under weaker hypotheses and different proof. Indeed, in Theorem 3.1 and Theorem 3.2 we have $\mu_{1} \equiv 1$ and $\hat{H}<0$ on $\{b\} \times(c, d)$ so that $(3.20)$ holds since $\hat{V}_{x}(b-, e) \leq 0$ for $e \in(c, d)$. Similarly, in Theorem 3.3 we have $\mu \equiv-1, \hat{V}_{y} \geq 0$ and $\hat{H}_{y} \geq \varepsilon>0$ on $[a, b] \times(c, d)$. Thus if (3.20) would not hold, then $\mu_{1} \hat{V}_{x}=-\hat{V}_{x}=-H$ on $\{b\} \times(c, d)$ and hence $\hat{V}_{x y}=\hat{H}_{y}$ on $\{b\} \times(c, d)$. By continuity up to $\{b\} \times(c, d)$ (obtained analogously to (3.16) upon applying $\partial_{y}$ in $(3.13)+(3.14)$ above) this implies that $\hat{V}_{y x}=\hat{V}_{x y} \geq \varepsilon / 2$ on $[a, b] \times(c, d)$ for $a<b$ sufficiently close to $b$. But then $\varepsilon(b-a) / 2 \leq \int_{a}^{b} \hat{V}_{y x}(x, e) d x=-V_{y}(a, e) \leq 0$ for $e \in(c, d)$ which is a contradiction. Thus (3.20) holds in this case as well and hence by Corollary 5 we can conclude that the optimal stopping boundary is continuous whenever increasing as concluded in Theorems 3.1-3.3. Note moreover that Corollary 5 includes numerous other cases that are not covered by those theorems.

3. Elliptic case. If $\operatorname{det}(\sigma) \neq 0$ (elliptic equation) then the infinitesimal generator of $Z=$ $(X, Y)$ solving $(2.2)-(2.4)$ is given by

$$
\mathbb{L}_{Z}=\alpha \partial_{x x}+2 \beta \partial_{x y}+\gamma \partial_{y y}+\mu_{1} \partial_{x}+\mu_{2} \partial_{y}
$$

where $\alpha>0, \beta \geq 0$ and $\gamma>0$ are given by (2.8) above. If $S$ is a subset of $\mathbb{R}^{2}$ then recalling that $C^{1}(S)$ denotes the family of continuous real-valued functions $F$ on $S$ such that $F_{x}$ and $F_{y}$ exist and are continuous on $S$, we let $C_{p}^{1}(S)$ denote the family of functions $F$ in $C^{1}(S)$ satisfying $F, F_{x}, F_{y} \in C_{p}(S)$ for $p>0$. Similarly, recalling that $C^{2}(S)$ denotes the family of continuous real-valued functions $F$ on $S$ such that $F_{x}, F_{y}, F_{x x}, F_{x y}, F_{y y}$ exist and are continuous on $S$, we let $C_{p}^{2}(S)$ denote the family of functions $F$ in $C^{2}(S)$ satisfying $F, F_{x}, F_{y}, F_{x x}, F_{x y}, F_{y y} \in C_{p}(S)$ for $p>0$.

Recalling Definitions 1 and 2 above the first main result of this section in the elliptic case can now be stated as follows (see also Corollary 18 below).

Theorem 7. Consider the optimal stopping problem (2.1) when $\operatorname{det}(\sigma) \neq 0$ upon assuming that it is well posed so that (2.14) is satisfied. Assume moreover that we have

$$
\alpha, \beta, \gamma, \mu_{1}, \mu_{2}, \lambda, \hat{H} \in C_{p}([a, b] \times[c, d])
$$

for some $p>0$ with some $a<b$ and $c<d$ such that $[a, b) \times[c, d] \subseteq C$ and $\{b\} \times[c, d] \subseteq D$, and that either of the two conditions is satisfied

$$
\begin{aligned}
& \hat{V}_{x x}(b-, e) \neq-\frac{\hat{H}(b, e)}{\alpha(b, e)} \\
& \hat{V}_{y y}(b-, e) \neq 0 \quad\left(\text { or } \hat{V}_{y}(b-, e) \neq 0\right)
\end{aligned}
$$


for at least one point $e \in(c, d)$. If the horizontal smooth fit holds on $\{b\} \times(c, d)$, then the optimal stopping boundary $\partial C$ has no discontinuity of the first kind at $b$.

Proof. 1. Setting $R:=[a, b] \times[c, d]$ we see from (3.22) that $\alpha, \gamma \in C([a, b] \times[c, d])$. Due to $\alpha$ and $\gamma$ being strictly positive, it is therefore clear that the differential operator $\mathbb{L}_{Z}-\lambda I$ is strictly elliptic on $R^{0}$ (in the sense that $(\sigma z, z) \geq k|z|^{2}$ for all $z \neq 0$ in $R^{0}$ with some $k>0$ given and fixed). Moreover, noting that an exterior sphere condition holds at every point $z \in \partial R$ (i.e. there exist $w \in R^{c}$ and $r>0$ such that $b(w, r) \subseteq R^{c}$ and $\left.z \in \partial(b(w, r))\right)$, and recalling that $\hat{V}$ is continuous on $R$, by the existence and uniqueness result for elliptic equations based on the a priori interior estimates of Schauder type [15, Theorem 6.13, p. 106] we can conclude using (3.22) that the boundary value problem

$$
\begin{aligned}
& \alpha \tilde{V}_{x x}+2 \beta \tilde{V}_{x y}+\gamma \tilde{V}_{y y}+\mu_{1} \tilde{V}_{x}+\mu_{2} \tilde{V}_{y}-\lambda \tilde{V}=-\hat{H} \text { on } R^{0} \\
& \tilde{V}=\hat{V} \quad \text { on } \partial R
\end{aligned}
$$

has a unique solution $\tilde{V}$ in $C_{p}^{2}\left(R^{0}\right) \cap C(R)$.

2. Letting $\tau_{1}=\inf \left\{t \geq 0 \mid Z_{t} \in \partial R\right\}$ denote the first entry time of $Z$ to $\partial R$, and using exactly the same arguments as in (3.15) above, we find that $\hat{V}=\tilde{V}$ on $R$ and hence $\hat{V}$ belongs to $C_{p}^{2}\left(R^{0}\right) \cap C(R)$.

3. Moreover, we claim that

$$
\hat{V} \in C_{p}^{2}\left(R^{1}\right)
$$

where we recall that $R^{1}=(a, b] \times(c, d)$. Indeed, this follows from the fact that the flat boundary portion $\{b\} \times(c, d)$ is the graph of a constant function which evidently belongs to $C_{p}^{2}((c, d))$, while $\hat{V}$ belongs to $C^{2}\left(R^{0}\right) \cap C(R)$, solves the boundary value problem $(3.25)+(3.26)$, and at the flat boundary portion $\{b\} \times(c, d)$ coincides with the zero function which evidently belongs to $C_{p}^{2}(R)$ (see $[15$, Lemma 6.18, p. 111]).

4. Summarising the facts derived above we know that $\hat{V}$ solves

$$
\alpha \hat{V}_{x x}+2 \beta \hat{V}_{x y}+\gamma \hat{V}_{y y}+\mu_{1} \hat{V}_{x}+\mu_{2} \hat{V}_{y}-\lambda \hat{V}=-\hat{H} \quad \text { on } R^{0}
$$

and belongs to $C_{p}^{2}\left(R^{1}\right) \cap C(R)$ as established in (3.27) above. Since $\hat{V}=0$ on $\{b\} \times(c, d)$ we see that $\hat{V}_{y}=\hat{V}_{y y}=0$ on $\{b\} \times(c, d)$ which contradicts (3.24) if satisfied. Moreover, if the horizontal smooth fit holds on $\{b\} \times(c, d)$, then $\hat{V}_{x}=0$ on $\{b\} \times(c, d)$ so that $\hat{V}_{x y}=0$ on $\{b\} \times(c, d)$ as well. Hence using the fact that $\hat{V} \in C^{2}\left(R^{1}\right)$ and taking any sequence $b_{n} \uparrow b$ as $n \rightarrow \infty$ we see that $\lim _{n \rightarrow \infty} \hat{V}\left(b_{n}, e\right)=\lim _{n \rightarrow \infty} \hat{V}_{y}\left(b_{n}, e\right)=\lim _{n \rightarrow \infty} \hat{V}_{y y}\left(b_{n}, e\right)=$ $\lim _{n \rightarrow \infty} \hat{V}_{x}\left(b_{n}, e\right)=\lim _{n \rightarrow \infty} \hat{V}_{x y}\left(b_{n}, e\right)=0$. Thus evaluating both sides in $(3.25)$ at $\left(b_{n}, e\right) \in$ $R^{0}$ and letting $n \rightarrow \infty$ we obtain $\alpha(b, e) \hat{V}_{x x}(b-, e)=-\hat{H}(b, e)$ which contradicts (3.23) if satisfied. Hence if either (3.23) or (3.24) holds then the optimal stopping boundary $\partial C$ cannot have a discontinuity of the first kind at $b$ as claimed.

Recalling the arguments following (2.13) above we know that there is no restriction in the setting of Theorem 7 to assume that $\hat{H} \leq 0$ on $\{b\} \times[c, d]$. We will now show that if $\hat{H}=0$ 
on $R=[a, b] \times[c, d]$ with $a<b$ sufficiently close to $b$ then the optimal stopping boundary cannot have a discontinuity of the first kind at $b$ (see also Corollary 19 below). This stands in sharp contrast with the parabolic case where this is possible (cf. [5, Remark 17]).

Corollary 8. Consider the optimal stopping problem (2.1) when $\operatorname{det}(\sigma) \neq 0$ upon assuming that it is well posed so that (2.14) is satisfied. Assume moreover that (3.22) holds for some $p>0$ and that $\hat{H} \geq 0$ on $[a, b] \times[c, d]$ with $a<b$ and $c<d$ such that $[a, b) \times[c, d] \subseteq C$ and $\{b\} \times[c, d] \subseteq \partial C \quad$ (so that $\hat{H}=0$ on $\{b\} \times[c, d]$ at least). If the horizontal smooth fit holds at $(b, e)$ for some $e \in(c, d)$, then the optimal stopping boundary $\partial C$ has no discontinuity of the first kind at $b$.

Proof. Proceeding as in the proof of Theorem 7 above we know from (3.28) that $\hat{V}$ belongs to $C^{2}\left(R^{0}\right)$ and solves $\mathbb{L}_{Z} \hat{V}-\lambda \hat{V}=-\hat{H}$ on $R^{0}$ where $\mathbb{L}_{Z}$ is given by (3.21) above and we recall that $R^{0}=(a, b) \times(c, d)$. Since $\alpha, \beta, \gamma, \mu_{1}, \mu_{2}, \lambda \in C(R)$ with $\alpha$ and $\gamma$ being strictly positive we see that the differential operator $\mathbb{L}_{Z}-\lambda I$ is uniformly elliptic on $R^{0}$ (in the sense that $k|z|^{2} \leq(\sigma z, z) \leq K|z|^{2}$ for all $z \neq 0$ in $R^{0}$ with some $0<k<K<\infty$ given and fixed). Since $\hat{H} \geq 0$ on $R^{0}$ it follows that $\mathbb{L}_{Z} \hat{V}-\lambda \hat{V} \leq 0$ on $R^{0}$. Moreover, we know that $\hat{V}$ is continuous at $(b, e) \in \partial R$ and satisfies $\hat{V}(x, y)>\hat{V}(b, e)=0$ for all $(x, y) \in R^{0}$. Finally, it is evident that an interior sphere condition holds at $z:=(b, e) \in \partial R$ (i.e. there exist $w \in R^{0}$ and $r>0$ such that $b(w, r) \subseteq R^{0}$ and $\left.z \in \partial(b(w, r))\right)$. These facts show that Hopf's boundary point lemma for elliptic equations (see e.g. [15, Lemma 3.4, p. 34]) is applicable in this setting and hence the outer normal derivative of $\hat{V}$ at $z=(b, e)$ must be strictly negative. In other words, we have $\hat{V}_{x}(b-, e)<0$ which contradicts the assumption that a horizontal smooth fit holds at $z=(b, e)$. Hence the optimal stopping boundary $\partial C$ cannot have a discontinuity of the first kind at $b$ as claimed.

Remark 9. The proof above shows that the second derivative conditions (3.23) and (3.24) are equivalent to each other when both the horizontal smooth fit $\hat{V}_{x}(b, e)=0$ and the vertical smooth fit $\hat{V}_{y}(b, e)=0$ hold. In view of (3.24) therefore the result of Theorem 7 tells us that the optimal stopping boundary $\partial C$ has no discontinuity of the first kind at $b$ whenever the 'proper' smooth fit holds in the vertical direction at $b$ (i.e. $\hat{V}_{y}(b, e)=0$ but $\hat{V}_{y y}(b, e) \neq 0$ ).

While the second derivative conditions (3.23) and (3.24) can be verified in some specific examples, establishing either of them may also be more challenging in general. For this reason it is desirable to obtain sufficient conditions expressed in terms of the first partial derivatives which are easier to verify. The following theorem derives such sufficient conditions under more regularity on the coefficients in (3.22) above (see also Corollary 20 below).

Theorem 10. Consider the optimal stopping problem (2.1) when $\operatorname{det}(\sigma) \neq 0$ upon assuming that it is well posed so that (2.14) is satisfied. Assume moreover that we have

$$
\alpha, \beta, \gamma, \mu_{1}, \mu_{2}, \lambda, \hat{H} \in C_{p}^{1}([a, b] \times[c, d])
$$

for some $p>0$ with some $a<b$ and $c<d$ such that $[a, b) \times[c, d] \subseteq C$ and $\{b\} \times[c, d] \subseteq D$, and that either of the two conditions is satisfied

$$
\partial_{y} \hat{V} \geq 0 \quad \text { on }(a, b) \times(c, d) \quad \& \quad \partial_{y}\left(\frac{\hat{H}}{\alpha}\right)>0 \quad \text { on }\{b\} \times[c, d]
$$




$$
\partial_{y} \hat{V} \leq 0 \quad \text { on }(a, b) \times(c, d) \quad \& \quad \partial_{y}\left(\frac{\hat{H}}{\alpha}\right)<0 \quad \text { on }\{b\} \times[c, d]
$$

If the horizontal smooth fit holds on $\{b\} \times(c, d)$, then the optimal stopping boundary $\partial C$ has no discontinuity of the first kind at $b$.

Proof. Proceeding as in the proof of Theorem 7 above we know that $\hat{V}$ belongs to $C_{p}^{2}\left(R^{0}\right) \cap C(R)$ and solves (3.28) on $R^{0}$. Due to (3.29) we can use the interior regularity theorem (see [15, Theorem 6.17, p. 109]) and conclude that $\hat{V}$ belongs to $C_{p}^{3}\left(R^{0}\right) \cap C(R)$. Moreover, we have established in the proof of Theorem 7 above that $\hat{V}$ belongs to $C_{p}^{2}\left(R^{1}\right)$ using the fact that the flat boundary portion $\{b\} \times(c, d)$ is the graph of a constant function which moreover belongs to $C_{p}^{3}((c, d))$, while $\hat{V}$ belongs to $C^{2}\left(R^{0}\right) \cap C(R)$, solves the boundary value problem $(3.25)+(3.26)$, and at the flat boundary portion $\{b\} \times(c, d)$ coincides with the zero function which thus belongs to $C_{p}^{3}(R)$. Strengthening the argument of [15, Lemma 6.18, p. 111] used in the proof of Theorem 7 and employing [15, Theorem 6.19, p. 111] instead (see also the remark following its proof) we can conclude that $\hat{V}$ belongs to $C_{p}^{3}\left(R^{1}\right)$. Taking the partial derivative $\partial_{y}$ on both sides of (3.28) we find that

$$
\begin{aligned}
\alpha\left(\hat{V}_{y}\right)_{x x}+2 \beta\left(\hat{V}_{y}\right)_{x y}+\gamma\left(\hat{V}_{y}\right)_{y y}= & -\hat{H}_{y}-\alpha_{y} \hat{V}_{x x}-\left(2 \beta_{y}+\mu_{1}\right) \hat{V}_{x y}-\left(\gamma_{y}+\mu_{2}\right) \hat{V}_{y y} \\
& -\left(\mu_{1}\right)_{y} \hat{V}_{x}-\left(\left(\mu_{2}\right)_{y}-\lambda\right) \hat{V}_{y}+\lambda_{y} \hat{V} \text { on } R^{0} .
\end{aligned}
$$

Setting $\tilde{\mathbb{L}}_{Z}=\alpha \partial_{x x}+2 \beta \partial_{x y}+\gamma \partial_{y y}$ and defining $\tilde{V}(x, y)=\hat{V}_{y}(x, y) \pm \varepsilon(x-b)^{2}$ for $(x, y) \in R^{0}$ with the plus sign if (3.30) holds and the minus sign if (3.31) holds, we see from (3.32) that $\tilde{V}$ belongs to $C^{2}\left(R^{0}\right)$ and solves

$$
\begin{aligned}
\tilde{\mathbb{L}}_{Z} \tilde{V}= & -\hat{H}_{y}-\alpha_{y} \hat{V}_{x x}-\left(2 \beta_{y}+\mu_{1}\right) \hat{V}_{x y}-\left(\gamma_{y}+\mu_{2}\right) \hat{V}_{y y} \\
& -\left(\mu_{1}\right)_{y} \hat{V}_{x}-\left(\left(\mu_{2}\right)_{y}-\lambda\right) \hat{V}_{y}+\lambda_{y} \hat{V} \pm 2 \varepsilon \alpha \text { on } R^{0} .
\end{aligned}
$$

Since $\alpha, \beta, \gamma \in C(R)$ with $\alpha$ and $\beta$ being strictly positive we see that the differential operator $\tilde{\mathbb{L}}_{Z}$ is uniformly elliptic on $R^{0}$. Recalling from the proof of Theorem 7 that $\hat{V}_{x x}(b-, y)=$ $-\hat{H}(b, y) / \alpha(b, y)$ and that $\hat{V}_{y}(b-, y)=\hat{V}_{y y}(b-, y)=\hat{V}_{x y}(b-, y)=0$ due to $\hat{V}(b-, y)=0$ with $\hat{V}_{x}(b-, y)=0$ by the horizontal smooth fit assumption for $y \in(c, d)$, we see that evaluating both sides of (3.33) at $\left(b_{n}, y\right)$ with $b_{n} \uparrow b$ as $n \rightarrow \infty$ the right-hand side in (3.33) tends to

$$
-\alpha(z) \partial_{y}\left(\frac{H}{\alpha}\right)(z) \pm 2 \varepsilon \alpha(z)
$$

where we set $z=(b, y)$ for $y \in(c, d)$. Since $z \mapsto-\alpha(z) \partial_{y}\left(\frac{H}{\alpha}\right)(z)$ is continuous on the compact set $\{b\} \times[c, d]$, we see that this function attains its strictly negative maximum on $\{b\} \times[c, d]$ when (3.30) holds and its strictly positive minimum on $\{b\} \times[c, d]$ when (3.31) holds. Choosing $\varepsilon>0$ sufficiently small we thus see by continuity that the entire expression in (3.34) remains either strictly negative on $\{b\} \times[c, d]$ when (3.30) holds or strictly positive on $\{b\} \times[c, d]$ when (3.31) holds. Choosing $a<b$ sufficiently close to $b$, and $[c, d]$ with $c<d$ sufficiently small, we see by continuity that the right-hand side in (3.33) remains either (strictly) negative on $(a, b) \times(c, d)$ when (3.30) holds or (strictly) positive on $(a, b) \times(c, d)$ when (3.31) holds. Recalling that $R^{0}=(a, b) \times(c, d)$ we therefore see from (3.33) that $\tilde{\mathbb{L}}_{Z} \tilde{V} \leq 0$ on $R^{0}$ when (3.30) holds and $\tilde{\mathbb{L}}_{Z} \tilde{V} \geq 0$ on $R^{0}$ when (3.31) holds. Moreover, fixing $e \in(c, d)$ 
we know that $\tilde{V}$ is continuous at $(b, e) \in \partial C$ and satisfies $\tilde{V}(x, y)>\tilde{V}(b, e)=0$ for all $(x, y) \in R^{0}$ when (3.30) holds and $\tilde{V}(x, y)<\tilde{V}(b, e)=0$ for all $(x, y) \in R^{0}$ when (3.31) holds. Finally, it is evident that an interior sphere condition holds at $(b, e) \in \partial C$. These facts show that Hopf's boundary point lemma for elliptic equations (see e.g. [15, Lemma 3.4, p. 34]) is applicable in this setting and hence the outer normal derivative of $\tilde{V}$ at $(b, e)$ must be strictly negative when (3.30) holds and must be strictly positive when (3.31) holds. In other words, we must either have $\tilde{V}_{x}(b-, e)=\hat{V}_{x y}(b-, e)<0$ or $\tilde{V}_{x}(b-, e)=\hat{V}_{x y}(b-, e)>0$ respectively. This conclusion, however, contradicts the fact that a horizontal smooth fit holds at $(b, e)$ so that $\hat{V}_{x y}(b-, e)=0$ in both cases due to $\hat{V} \in C^{2}\left(R^{1}\right)$. Hence the optimal stopping boundary $\partial C$ cannot have a discontinuity of the first kind at $b$ as claimed.

Remark 11. Note that the result of Theorem 10 implies continuity of the optimal stopping boundary studied in the simpler setting of [8, pp. 15-19] where an ad-hoc argument based on the Feynman-Kac formula was used instead.

The monotonicity conditions (3.30) and (3.31) are usually verifiable in specific examples unless $A \not \equiv 0$ in (2.10) when such a verification may be more challenging (due to the presence of local times). In the latter case it may be possible to exploit the (local) convexity/concavity of the value function instead. The following theorem accomplishes that aim by extending the rationale of Theorem 10 to the second partial derivatives (in the vertical direction) under additional regularity on the coefficients in (3.29) above (see also Corollary 21 below).

Theorem 12. Consider the optimal stopping problem (2.1) when $\operatorname{det}(\sigma) \neq 0$ upon assuming that it is well posed so that (2.14) is satisfied. Assume moreover that we have

$$
\alpha, \beta, \gamma, \mu_{1}, \mu_{2}, \lambda, \hat{H} \in C_{p}^{2}([a, b] \times[c, d])
$$

for some $p>0$ with some $a<b$ and $c<d$ such that $[a, b) \times[c, d] \subseteq C$ and $\{b\} \times[c, d] \subseteq D$, and that either of the two conditions is satisfied

$$
\begin{aligned}
& \partial_{y y} \hat{V} \geq 0 \quad \text { on }(a, b) \times(c, d) \quad \& \quad \partial_{y y}\left(\frac{\hat{H}}{\alpha}\right)>0 \text { on }\{b\} \times[c, d] \\
& \partial_{y y} \hat{V} \leq 0 \quad \text { on }(a, b) \times(c, d) \quad \& \quad \partial_{y y}\left(\frac{\hat{H}}{\alpha}\right)<0 \quad \text { on }\{b\} \times[c, d] .
\end{aligned}
$$

If the horizontal smooth fit holds on $\{b\} \times(c, d)$, then the optimal stopping boundary $\partial C$ has no discontinuity of the first kind at $b$.

Proof. Arguing in exactly the same way as in the proof of Theorem 10 upon using (3.35) in place of (3.29) we can conclude that $\hat{V}$ solves (3.28) and belongs to $C_{p}^{4}\left(R^{1}\right)$. Taking the partial derivative $\partial_{y}$ on both sides of $(3.32)$ we find that

$$
\begin{aligned}
\alpha\left(\hat{V}_{y y}\right)_{x x} & +2 \beta\left(\hat{V}_{y y}\right)_{x y}+\gamma\left(\hat{V}_{y y}\right)_{y y}=-\hat{H}_{y y}-\alpha_{y y} \hat{V}_{x x}-2 \alpha_{y}\left(\hat{V}_{x x}\right)_{y}-\left(4 \beta_{y}+\mu_{1}\right) \hat{V}_{x y y} \\
& -\left(2 \gamma_{y}+\mu_{2}\right) \hat{V}_{y y y}-\left(\gamma_{y y}+2\left(\mu_{2}\right)_{y}-\lambda\right) \hat{V}_{y y}-2\left(\beta_{y y}+\left(\mu_{1}\right)_{y}\right) \hat{V}_{x y}-\left(\mu_{1}\right)_{y y} \hat{V}_{x} \\
& -\left(\left(\mu_{2}\right)_{y y}-2 \lambda_{y}\right) \hat{V}_{y}+\lambda_{y y} \hat{V} \text { on } R^{0} .
\end{aligned}
$$

Setting $\tilde{\mathbb{L}}_{Z}=\alpha \partial_{x x}+2 \beta \partial_{x y}+\gamma \partial_{y y}$ and defining $\tilde{V}(x, y)=\hat{V}_{y y}(x, y) \pm \varepsilon(x-b)^{2}$ for $(x, y) \in R^{0}$ with the plus sign if (3.36) holds and the minus sign if (3.37) holds, we see from (3.38) that $\tilde{V}$ 
belongs to $C^{2}\left(R^{0}\right)$ and solves

$$
\begin{aligned}
\tilde{\mathbb{L}}_{Z} \tilde{V}= & -\hat{H}_{y y}-\alpha_{y y} \hat{V}_{x x}-2 \alpha_{y}\left(\hat{V}_{x x}\right)_{y}-\left(4 \beta_{y}+\mu_{1}\right) \hat{V}_{x y y}-\left(2 \gamma_{y}+\mu_{2}\right) \hat{V}_{y y y} \\
& -\left(\gamma_{y y}+2\left(\mu_{2}\right)_{y}-\lambda\right) \hat{V}_{y y}-2\left(\beta_{y y}+\left(\mu_{1}\right)_{y}\right) \hat{V}_{x y}-\left(\mu_{1}\right)_{y y} \hat{V}_{x} \\
& -\left(\left(\mu_{2}\right)_{y y}-2 \lambda_{y}\right) \hat{V}_{y}+\lambda_{y y} \hat{V} \pm 2 \varepsilon \alpha \text { on } R^{0} .
\end{aligned}
$$

Arguing in exactly the same way as following (3.33) above, we see that evaluating both sides of (3.39) at $\left(b_{n}, y\right)$ with $b_{n} \uparrow b$ as $n \rightarrow \infty$ the right-hand side in (3.33) tends to

$$
-\alpha(z) \partial_{y y}\left(\frac{H}{\alpha}\right)(z) \pm 2 \varepsilon \alpha(z)
$$

where we set $z=(b, y)$ for $y \in(c, d)$. Proceeding then in exactly the same way as following (3.34) above, we find by Hopf's boundary point lemma that either $\tilde{V}_{x}(b-, e)=\hat{V}_{x y y}(b-, e)<$ 0 if $(3.36)$ holds or $\tilde{V}_{x}(b-, e)=\hat{V}_{x y y}(b-, e)>0$ if $(3.37)$ holds. This conclusion however contradicts the fact that a horizontal smooth fit holds at $(b, e)$ so that $\hat{V}_{x y y}(b-, e)=0$ in both cases due to $\hat{V} \in C^{3}\left(R^{1}\right)$. Hence the optimal stopping boundary $\partial C$ cannot have a discontinuity of the first kind at $b$ as claimed.

Remark 13. The results of Theorem 10 and Theorem 12 do not exhaust all possibilities and further refinements of the main argument in the proof are possible in specific examples. For instance, if $\partial_{y}(\hat{H} / \alpha)$ in $(3.30) /(3.31)$ or $\partial_{y y}(\hat{H} / \alpha)$ in $(3.36) /(3.37)$ equals zero, and all the terms but the first two on the right-hand side of (3.32) or all the terms but the first three on the right-hand side of (3.38) produce a non-positive/non-negative sum, then on careful examination of the proofs above (by letting $\varepsilon$ be zero) one sees that the conclusion of Theorem 10 or Theorem 12 is still valid if one has that $\partial_{y} \hat{V}$ in $(3.30) /(3.31)$ or $\partial_{y y} \hat{V}$ in $(3.36) /(3.37)$ is strictly positive/strictly negative respectively (note that this also leads to extensions of Corollary 20 and Corollary 21 below using Theorem 12 below).

\section{Regularity implies smooth fit}

Consider the optimal stopping problem (2.1) upon assuming that it is well posed as discussed in Section 2 above. In this section we show that a horizontal smooth fit between the value function $V$ and the gain function $G$ is satisfied over the flat portion of the optimal stopping boundary arising from any of its hypothesised jumps. This fact holds in the elliptic case $(\operatorname{det}(\sigma) \neq 0)$ while the parabolic case $(\operatorname{det}(\sigma)=0)$ is settled by Corollary 5 as we show first.

1. Parabolic case. If $\operatorname{det}(\sigma)=0$ (parabolic equation) and $\mu_{1}>0$ at $(b, e) \in \partial C$ then as stated in Remark 4 we know by (3.20) and the result of Corollary 5 that the optimal stopping boundary $\partial C$ has no discontinuity of the first kind at $b$. The same argument also applies to the case $\mu_{1}=0$ on $\{b\} \times[c, d]$ whenever $\hat{H} \neq 0$ at one point form $\{b\} \times[c, d]$. We will now show, as indicated in Remark 4, that the horizontal smooth fit can break down at $(b, e) \in \partial C$ when (i) $\mu_{1}(b, e)<0$ and (ii) the optimal stopping boundary $\partial C$ has a discontinuity of the first kind at $b$. In other words, in the absence of a horizontal smooth fit it is possible that the optimal stopping boundary develops discontinuities of the first kind, thus confirming that the two phenomena are intimately related. 
Example 14. Consider the optimal stopping problem

$$
V=\sup _{0 \leq \tau \leq 1} \mathrm{E}\left[\left|B_{\tau}\right|^{2}-\sqrt{2 \tau}\right]
$$

where the supremum is taken over stopping times $\tau$ of a standard Brownian motion $B$. (Note that $\left(t, B_{t}\right)=\left(X_{t}, Y_{t}\right)$ for $t \in[0,1]$ in the notation of Section 2 above.) Enabling the process to start at arbitrary points, and making use of the optional sampling theorem, we find that

$$
\begin{aligned}
V(t, x) & =\sup _{0 \leq \tau \leq 1-t} \mathrm{E}\left[\left|x+B_{\tau}\right|^{2}-\sqrt{2(t+\tau)}\right] \\
& =x^{2}-t+\sup _{0 \leq \tau \leq 1-t} \mathrm{E}[(t+\tau)-\sqrt{2(t+\tau)}]=: x^{2}-t+\tilde{V}(t)
\end{aligned}
$$

for $(t, x) \in[0,1] \times \mathbb{R}$. Defining the gain function of $\tilde{V}$ by $\tilde{G}(t)=t-\sqrt{2 t}$ for $t \in[0,1]$, we see that $\tilde{G}$ takes value 0 at 0 , is strictly decreasing on $(0,1 / 2)$, is strictly increasing on $(1 / 2,1)$, and satisfies $\tilde{G}(b)=\tilde{G}(1)=1-\sqrt{2}<0$ where we set $b:=3-2 \sqrt{2} \in(0,1 / 2)$. This shows that the stopping set $D$ for $\tilde{V}$ (and thus $V$ too) equals $[0, b] \times \mathbb{R}$ and the continuation set $C$ for $\tilde{V}$ (and thus $V$ too) equals $(b, 1) \times \mathbb{R}$. Note that this is the parabolic setting considered in Sections 2 and 3 above when $\mu_{1}<0$ (as the process moves away from the optimal stopping set). It follows from the descriptions of $C$ and $D$ that $\tilde{V}(t)=t-\sqrt{2 t}$ if $0 \leq t \leq b$ and $\tilde{V}(t)=1-\sqrt{2}$ if $b \leq t \leq 1$. Inserting this back into (4.2), and noting that the gain function of $V$ is given by $G(t, x)=x^{2}-\sqrt{2 t}$ for $(t, x) \in[0,1] \times \mathbb{R}$, we find that

$$
-1=V_{t}(b+, x) \neq G_{t}(b, x)=-\frac{1}{\sqrt{2 b}}
$$

for $x \in \mathbb{R}$. This shows that the horizontal smooth fit breaks down at the vertical flat portion of the optimal stopping boundary as claimed.

2. Elliptic case. If $\operatorname{det}(\sigma) \neq 0$ (elliptic equation) then a horizontal smooth fit between the value function $V$ and the gain function $G$ is satisfied over the flat portion of the optimal stopping boundary whenever proper in the sense described below. The first step in the proof consists of showing that the first component $X$ of the process $Z=(X, Y)$ develops a nontrivial local time having a square-root growth at zero after starting at the flat boundary.

Recall that the (semimartingale) local time of $X$ at $b$ in $\mathbb{R}$ is defined by

$$
\ell_{t}^{b}(X)=\mathrm{P}-\lim _{\varepsilon \downarrow 0} \frac{1}{2 \varepsilon} \int_{0}^{t} I\left(b-\varepsilon<X_{s}<b+\varepsilon\right) d\langle X, X\rangle_{s}
$$

for $t \geq 0$ where the convergence takes place in probability and $\langle X, X\rangle$ denotes the quadratic variation process of $X$ (cf. [23, Chapter 6]). In addition to $R=[a, b] \times[c, d]$ for $a<b$ and $c<d$ in $\mathbb{R}$ we also set $R_{1}=\left[a, b_{1}\right] \times[c, d]$ for $a<b<b_{1}$ in $\mathbb{R}$. Throughout this section we let $\rho_{1}$ denote the first exit time of $Z=(X, Y)$ from $R_{1}$ given by

$$
\rho_{1}=\inf \left\{t \geq 0 \mid Z_{t}=\left(X_{t}, Y_{t}\right) \notin R_{1}\right\}
$$

under $\mathrm{P}_{b, e}$ with $e \in(c, d)$ given and fixed. 
Lemma 15. We have

$$
\mathrm{E}_{b, e}\left[\ell_{t \wedge \rho_{1}}^{b}(X)\right] \geq c \sqrt{t}
$$

for all $t \in\left(0, t_{1}\right)$ with some $c>0$ and $t_{1}>0$.

Proof. Without loss of generality we may assume that $b=e=0$ and write $\mathrm{E}$ in place of $\mathrm{E}_{b, e}$ throughout. By the Itô-Tanaka formula (cf. [23, p. 223]) using (2.2) we get

$$
\left|X_{t}\right|=\int_{0}^{t} \operatorname{sign}\left(X_{s}\right) d X_{s}+\ell_{t}^{0}(X)=A_{t}+M_{t}+\ell_{t}^{0}(X)
$$

where $A_{t}=\int_{0}^{t} \operatorname{sign}\left(X_{s}\right) \mu_{1}\left(Z_{s}\right) d s$ is a continuous process of bounded variation and $M_{t}=$ $\int_{0}^{t} \operatorname{sign}\left(X_{s}\right) \sigma_{11}\left(Z_{s}\right) d B_{s}^{1}+\int_{0}^{t} \operatorname{sign}\left(X_{s}\right) \sigma_{12}\left(Z_{s}\right) d B_{s}^{2}$ is a continuous local martingale for $t \geq 0$. By Skorokhod's lemma (cf. [23, p. 239]) we can conclude from (4.7) that

$$
\ell_{t}^{0}(X)=\sup _{0 \leq s \leq t}\left(-A_{s}-M_{s}\right)
$$

for $t \geq 0$. Setting $\bar{A}_{t}:=\int_{0}^{t}\left|\mu_{1}\left(Z_{s}\right)\right| d s$ and using that $\mu_{1}$ is continuous on the compact set $R_{1}$ we see from (4.8) that

$$
\ell_{t \wedge \rho_{1}}^{0}(X)=\sup _{0 \leq s \leq t \wedge \rho_{1}}\left(-A_{s}-M_{s}\right) \geq-\bar{A}_{t \wedge \rho_{1}}+\sup _{0 \leq s \leq t \wedge \rho_{1}}\left(-M_{s}\right) \geq-c_{1} t+\sup _{0 \leq s \leq t \wedge \rho_{1}}\left(-M_{s}\right)
$$

for $t \geq 0$ with some $c_{1}>0$. By the Dambis-Dubins-Schwarz theorem (cf. [23, pp. 181-182]) we know that the continuous local martingale $-M$ can be represented as a time-changed standard Brownian motion $B$ in the sense that $-M_{t}=B_{\langle M, M\rangle_{t}}$ for $t \geq 0$. Moreover, since $\sigma_{11}^{2}+\sigma_{12}^{2}>0$ is continuous on the compact set $R_{1}$ it follows that

$$
\langle M, M\rangle_{t \wedge \rho_{1}}=\int_{0}^{t \wedge \rho_{1}}\left(\sigma_{11}^{2}\left(Z_{s}\right)+\sigma_{12}^{2}\left(Z_{s}\right)\right) d s \geq c\left(t \wedge \rho_{1}\right)
$$

for $t \geq 0$ with some $c>0$. This shows that

$$
\begin{aligned}
\sup _{0 \leq s \leq t \wedge \rho_{1}}\left(-M_{s}\right) & =\sup _{0 \leq s \leq t \wedge \rho_{1}} B_{\langle M, M\rangle_{s}}=\sup _{0 \leq s \leq\langle M, M\rangle_{t \wedge \rho_{1}}} B_{s} \geq \sup _{0 \leq s \leq c\left(t \wedge \rho_{1}\right)} B_{s} \\
& \geq\left(\sup _{0 \leq s \leq c t} B_{s}\right) I\left(t<\rho_{1}\right)=\sup _{0 \leq s \leq c t} B_{s}-\left(\sup _{0 \leq s \leq c t} B_{s}\right) I\left(\rho_{1} \leq t\right)
\end{aligned}
$$

for $t \geq 0$. Using that $\sup _{0 \leq s \leq c t} B_{s} \sim\left|B_{c t}\right| \sim \sqrt{c t}\left|B_{1}\right|$ and taking expectations in (4.9) and (4.11) we find that

$$
\mathrm{E}\left[\ell_{t \wedge \rho_{1}}^{0}(X)\right] \geq-c_{1} t+c_{2} \sqrt{t}-\mathrm{E}\left[\left(\sup _{0 \leq s \leq c t} B_{s}\right) I\left(\rho_{1} \leq t\right)\right]
$$

for $t \geq 0$ with $c_{1}>0$ and $c_{2}=\sqrt{2 c / \pi}>0$. Finally, by the Hölder inequality we find that

$$
\mathrm{E}\left[\left(\sup _{0 \leq s \leq c t} B_{s}\right) I\left(\rho_{1} \leq t\right)\right] \leq \sqrt{\mathrm{E}\left(\sup _{0 \leq s \leq c t} B_{s}\right)^{2}} \sqrt{\mathrm{P}\left(\rho_{1} \leq t\right)}=\sqrt{c t} \sqrt{\mathrm{P}\left(\rho_{1} \leq t\right)}
$$


for $t \geq 0$. Combining (4.12) and (4.13), and using that $\mathrm{P}\left(\rho_{1} \leq t\right) \rightarrow 0$ as $t \downarrow 0$, we obtain

$$
\liminf _{t \downarrow 0} \frac{1}{\sqrt{t}} \mathrm{E}\left[\ell_{t \wedge \rho_{1}}^{0}(X)\right] \geq c_{2}>0 .
$$

This establishes (4.6) and completes the proof.

Remark 16. Note that some precision is needed in the proof (see (4.11) above) to establish the inequality (4.6) since the non-modulus Burkholder-Davis-Gundy inequalities (to be applied to (4.8) or its time-changed version) generally break down for the power one (see [18, p. 540]) while we wish to impose no lower or upper bound on $\sigma_{11}^{2}+\sigma_{12}^{2}$ globally (thus going beyond uniformly elliptic PDEs). Note also that the known (Burkholder-Davis-Gundy type) inequalities for the semimartingale local times usually contain another supremum (over all $b$ ) under the expectation sign in (4.6) (see [1]). The lower bound established in (4.6) is of a different kind and cannot be derived from these maximal inequalities.

Theorem 17. Consider the optimal stopping problem (2.1) when $\operatorname{det}(\sigma) \neq 0$ upon assuming that it is well posed so that (2.14) is satisfied. Assume moreover that (3.22) holds for some $p>0$ and that the optimal stopping boundary $\partial C$ has a discontinuity of the first kind at $b \in \mathbb{R}$ in the sense that $[a, b) \times[c, d] \subseteq C$ and $\left[b, b_{1}\right] \times[c, d] \subseteq D$ for some $a<b$ and $b_{1}>b$ sufficiently close to $b$ and some sufficiently small $[c, d]$ with $c<d$. Then the horizontal smooth fit holds at $(b, e)$ for every $e \in(c, d)$.

Proof. 1. We have seen in the proof of Theorem 7 that under (3.22) the value function $\hat{V}$ belongs to $C^{2}\left(R^{1}\right)$ where $R^{1}=(a, b] \times(c, d)$. Moreover, from $(2.11)$ we know that $\hat{V}$ equals zero on $R^{2}:=\left[b, b_{1}\right) \times(c, d)$ and thus belongs to $C^{2}\left(R^{2}\right)$. It follows therefore that the change-of-variable formula with local time on surfaces [21, Equation (2.6)] is applicable to $e^{\Lambda} \hat{V}(Z)$ stopped at $\rho_{1}$ where we recall that $Z=(X, Y)$ and $\rho_{1}$ is defined by (4.5) above with $R_{1}=\left[a, b_{1}\right] \times[c, d]$. Moreover, since $\sigma_{11}^{2}+\sigma_{12}^{2}>0$ is continuous and therefore bounded below by a strictly positive constant on the compact set $R_{1}$, we see from the existence of the local time in (4.4) that $Z=(X, Y)$ stopped at $\rho_{1}$ does not spend any time on $\{b\} \times(c, d)$ in the sense that $\mathrm{E}_{b, e}\left[\int_{0}^{\rho_{1}} I\left(X_{s}=b\right) d s\right]=0$ for any $e \in(c, d)$. This shows that the first three terms in [21, Equation (2.6)] reduce to the classical Itô terms so that after regrouping we obtain

$$
\begin{aligned}
e^{-\Lambda_{t \wedge \rho_{1}} \hat{V}\left(Z_{t \wedge \rho_{1}}\right)=} & \hat{V}(z)+\int_{0}^{t \wedge \rho_{1}} e^{-\Lambda_{s}}\left(\mathbb{L}_{Z} \hat{V}-\lambda \hat{V}\right)\left(Z_{s}\right) d s+M_{t \wedge \rho_{1}} \\
& +\int_{0}^{t \wedge \rho_{1}} e^{-\Lambda_{s}}\left[\hat{V}_{x}\left(b+, Y_{s}\right)-\hat{V}_{x}\left(b-, Y_{s}\right)\right] d \ell_{s}^{b}(X)
\end{aligned}
$$

where $M_{t \wedge \rho_{1}}=\int_{0}^{t \wedge \rho_{1}} e^{-\Lambda_{s}} \hat{V}_{x}\left(Z_{s}\right) \sigma_{11}\left(Z_{s}\right) d B_{s}^{1}+\int_{0}^{t \wedge \rho_{1}} e^{-\Lambda_{s}} \hat{V}_{x}\left(Z_{s}\right) \sigma_{12}\left(Z_{s}\right) d B_{s}^{2}$ is a continuous (local) martingale for $t \geq 0$ and $z=(b, e) \in \partial C$ is given and fixed for some $e \in(c, d)$. Taking $\mathrm{E}_{z}$ on both sides in (4.15), upon recalling that $\hat{V}$ solves (3.13) on $R^{0} \subseteq C$ and equals zero on $R^{2} \subseteq D$, we find that

$$
\begin{aligned}
\mathrm{E}_{z}\left[e^{\left.-\Lambda_{t \wedge \rho_{1}} \hat{V}\left(Z_{t \wedge \rho_{1}}\right)\right]=}\right. & -\mathrm{E}_{z}\left[\int_{0}^{t \wedge \rho_{1}} e^{-\Lambda_{s}} \hat{H}\left(Z_{s}\right) I\left(Z_{s} \in C\right) d s\right] \\
& +\mathrm{E}_{z}\left[\int_{0}^{t \wedge \rho_{1}} e^{-\Lambda_{s}} \Delta \hat{V}_{x}\left(b, Y_{s}\right) d \ell_{s}^{b}(X)\right]
\end{aligned}
$$


where we set $\Delta \hat{V}_{x}\left(b, Y_{s}\right):=\hat{V}_{x}\left(b+, Y_{s}\right)-\hat{V}_{x}\left(b-, Y_{s}\right)=-\hat{V}_{x}\left(b-, Y_{s}\right) \geq 0$ for $0 \leq s \leq t$.

2. Returning back to the definition of $\hat{V}$ in (2.10), we may recall from the discussion following (2.8) that the process $A$ takes the form

$$
A_{t}=\sum_{i=1}^{n} \int_{0}^{t} e^{-\Lambda_{s}} J_{i}\left(Z_{s}\right) d L_{s}^{i}
$$

for $t \geq 0$ where $J_{i}$ equals either $\Delta G_{x}$ or $\Delta G_{y}$ and $L^{i}$ equals either $\ell^{b_{i}}(X)$ or $\ell^{b_{i}}(Y)$ respectively upon letting $b_{i}$ denote the continuous semimartingale curves off which $G$ is $C^{2}$ (see [21, Remark 2.2] for further details). Recall that if $G$ is $C^{2}$ globally then $A$ equals zero. Using (2.10) and applying the strong Markov property of $Z$ at $t \wedge \rho_{1}$ we see that

$$
\begin{aligned}
& e^{-\Lambda_{t \wedge \rho_{1}}} \hat{V}\left(Z_{t \wedge \rho_{1}}\right)=e^{-\Lambda_{t \wedge \rho_{1}}} \mathrm{E}_{Z_{t \wedge \rho_{1}}}\left(\int_{0}^{\tau_{D}} e^{-\Lambda_{s}} \hat{H}\left(Z_{s}\right) d s+\sum_{i=1}^{n} \int_{0}^{\tau_{D}} e^{-\Lambda_{s}} J_{i}\left(Z_{s}\right) d L_{s}^{i}\right) \\
& =\mathrm{E}_{z}\left[\int_{0}^{t \wedge \rho_{1}+\tau_{D} \circ \theta_{t \wedge \rho_{1}}} e^{-\Lambda_{s}} \hat{H}\left(Z_{s}\right) d s+\sum_{i=1}^{n} \int_{0}^{t \wedge \rho_{1}+\tau_{D} \diamond \theta_{t \wedge \rho_{1}}} e^{-\Lambda_{s}} J_{i}\left(Z_{s}\right) d L_{s}^{i} \mid \mathcal{F}_{t \wedge \rho_{1}}^{Z}\right) \\
& \quad-\int_{0}^{t \wedge \rho_{1}} e^{-\Lambda_{s}} \hat{H}\left(Z_{s}\right) d s-\sum_{i=1}^{n} \int_{0}^{t \wedge \rho_{1}} e^{-\Lambda_{s}} J_{i}\left(Z_{s}\right) d L_{s}^{i}
\end{aligned}
$$

for $t \geq 0$ where $\theta$ denotes the shift operator. Taking $\mathrm{E}_{z}$ on both sides we get

$$
\begin{aligned}
\mathrm{E}_{z}\left[e^{\left.-\Lambda_{t \wedge \rho_{1}} \hat{V}\left(Z_{t \wedge \rho_{1}}\right)\right]=}\right. & \mathrm{E}_{z}\left[\int_{0}^{t \wedge \rho_{1}+\tau_{D} \circ \theta_{t \wedge \rho_{1}}} e^{-\Lambda_{s}} \hat{H}\left(Z_{s}\right) d s+A_{t \wedge \rho_{1}+\tau_{D} \circ \theta_{t \wedge \rho_{1}}}\right] \\
& -\mathrm{E}_{z}\left[\int_{0}^{t \wedge \rho_{1}} e^{-\Lambda_{s}} \hat{H}\left(Z_{s}\right) d s\right] \leq-\mathrm{E}_{z}\left[\int_{0}^{t \wedge \rho_{1}} e^{-\Lambda_{s}} \hat{H}\left(Z_{s}\right) d s\right]
\end{aligned}
$$

for $t \geq 0$ where the equality follows since the final term in (4.18) can be made zero (by narrowing $R_{1}$ if needed) and the inequality follows due to $z \in \partial C$ so that $\hat{V}(z)=0$ while $t \wedge \rho_{1}+\tau_{D} \circ \theta_{t \wedge \rho_{1}}$ is a stopping time (not necessarily optimal).

3. Combining (4.16) and (4.19) we obtain

$$
\mathrm{E}_{z}\left[\int_{0}^{t \wedge \rho_{1}} e^{-\Lambda_{s}} \Delta \hat{V}_{x}\left(b, Y_{s}\right) d \ell_{s}^{b}(X)\right] \leq \mathrm{E}_{z}\left[\int_{0}^{t \wedge \rho_{1}} e^{-\Lambda_{s}}(-\hat{H})\left(Z_{s}\right) I\left(Z_{s} \in D\right) d s\right]
$$

for $t \geq 0$. If the horizontal smooth fit would not hold at $z=(b, e)$ then by continuity of $\hat{V}_{x}$ (and $\lambda$ ) on the compact set $R^{1}$ (and narrowing $R^{1}$ if needed) we see that $e^{-\Lambda_{s}} \Delta \hat{V}_{x}\left(b, Y_{s}\right) \geq$ $c_{1}>0$ for $0 \leq s \leq t \wedge \rho_{1}$ with $c_{1}>0$. Similarly, by continuity of $\hat{H}$ on $R^{1}$ we see that $(-\hat{H})\left(Z_{s}\right) \leq c_{2}$ for $0 \leq s \leq t \wedge \rho_{1}$ with $c_{2}>0$. Combining these facts in (4.20) we get

$$
c_{1} \mathrm{E}_{z}\left[\ell_{t}^{b}(X)\right] \leq c_{2} t
$$

for $t \geq 0$. This conclusion contradicts the inequality (4.6) established in Lemma 15 above and thus the horizontal smooth fit must hold on $\{b\} \times(c, d)$ as claimed. 
Note that the hypotheses in Theorem 17 differ from the hypotheses of Theorem 7 where a non-trivial rectangle to the right from the boundary point is not assumed to be contained in the stopping set. Adding this hypothesis implies the horizontal smooth fit and therefore can replace it. The same argument applies in the setting of Corollary 8, Theorem 10 and Theorem 12. In this way we obtain the following combined results.

Corollary 18. Consider the optimal stopping problem (2.1) when $\operatorname{det}(\sigma) \neq 0$ upon assuming that it is well posed so that (2.14) is satisfied. Assume moreover that we have

$$
\alpha, \beta, \gamma, \mu_{1}, \mu_{2}, \lambda, \hat{H} \in C_{p}([a, b] \times[c, d])
$$

for some $p>0$ with some $a<b$ and $c<d$ such that $[a, b) \times[c, d] \subseteq C$ and $\{b\} \times[c, d] \subseteq D$, and that either of the two conditions is satisfied

$$
\begin{aligned}
& \hat{V}_{x x}(b-, e) \neq-\frac{\hat{H}(b, e)}{\alpha(b, e)} \\
& \hat{V}_{y y}(b-, e) \neq 0 \quad\left(\text { or } \hat{V}_{y}(b-, e) \neq 0\right)
\end{aligned}
$$

for at least one point $e \in(c, d)$. If $\left[b, b_{1}\right] \times[c, d]$ is contained in $D$ for some $b_{1}>b$, then the optimal stopping boundary $\partial C$ has no discontinuity of the first kind at $b$.

Proof. This follows by combining the results of Theorem 7 and Theorem 17 .

Corollary 19. Consider the optimal stopping problem (2.1) when $\operatorname{det}(\sigma) \neq 0$ upon assuming that it is well posed so that (2.14) is satisfied. Assume moreover that (3.22) holds for some $p>0$ and that $\hat{H} \geq 0$ on $[a, b] \times[c, d]$ with $a<b$ and $c<d$ such that $[a, b) \times[c, d] \subseteq C$ and $\{b\} \times[c, d] \subseteq \partial C$. If $\left[b, b_{1}\right] \times[c, d]$ is contained in $D$ for some $b_{1}>b$, then the optimal stopping boundary $\partial C$ has no discontinuity of the first kind at $b$.

Proof. This follows by combining the results of Corollary 8 and Theorem 17.

Corollary 20. Consider the optimal stopping problem (2.1) when $\operatorname{det}(\sigma) \neq 0$ upon assuming that it is well posed so that (2.14) is satisfied. Assume moreover that we have

$$
\alpha, \beta, \gamma, \mu_{1}, \mu_{2}, \lambda, \hat{H} \in C_{p}^{1}([a, b] \times[c, d])
$$

for some $p>0$ with some $a<b$ and $c<d$ such that $[a, b) \times[c, d] \subseteq C$ and $\{b\} \times[c, d] \subseteq D$, and that either of the two conditions is satisfied

$$
\begin{array}{llll}
\partial_{y} \hat{V} \geq 0 & \text { on }(a, b) \times(c, d) \quad \& \quad \partial_{y}\left(\frac{\hat{H}}{\alpha}\right)>0 & \text { on } & \{b\} \times[c, d] \\
\partial_{y} \hat{V} \leq 0 & \text { on }(a, b) \times(c, d) \quad \& \quad \partial_{y}\left(\frac{\hat{H}}{\alpha}\right)<0 & \text { on }\{b\} \times[c, d] .
\end{array}
$$

If $\left[b, b_{1}\right] \times[c, d]$ is contained in $D$ for some $b_{1}>b$, then the optimal stopping boundary $\partial C$ has no discontinuity of the first kind at $b$.

Proof. This follows by combining the results of Theorem 10 and Theorem 17 . 
Corollary 21. Consider the optimal stopping problem (2.1) when $\operatorname{det}(\sigma) \neq 0$ upon assuming that it is well posed so that (2.14) is satisfied. Assume moreover that we have

$$
\alpha, \beta, \gamma, \mu_{1}, \mu_{2}, \lambda, \hat{H} \in C_{p}^{2}([a, b] \times[c, d])
$$

for some $p>0$ with some $a<b$ and $c<d$ such that $[a, b) \times[c, d] \subseteq C$ and $\{b\} \times[c, d] \subseteq D$, and that either of the two conditions is satisfied

$$
\begin{aligned}
& \partial_{y y} \hat{V} \geq 0 \quad \text { on }(a, b) \times(c, d) \quad \& \quad \partial_{y y}\left(\frac{\hat{H}}{\alpha}\right)>0 \quad \text { on }\{b\} \times[c, d] \\
& \partial_{y y} \hat{V} \leq 0 \quad \text { on }(a, b) \times(c, d) \quad \& \quad \partial_{y y}\left(\frac{\hat{H}}{\alpha}\right)<0 \quad \text { on }\{b\} \times[c, d] .
\end{aligned}
$$

If $\left[b, b_{1}\right] \times[c, d]$ is contained in $D$ for some $b_{1}>b$, then the optimal stopping boundary $\partial C$ has no discontinuity of the first kind at $b$.

Proof. This follows by combining the results of Theorem 12 and Theorem 17.

Acknowledgements. The author gratefully acknowledges financial support from Rice University, Houston, USA where the present paper was completed (May 2018). The author is indebted to Philip Ernst for the superb hospitality and insightful discussions.

\section{References}

[1] Barlow, M. T. and Yor, M. (1981). (Semi-) martingale inequalities and local times. Z. Wahrsch. Verw. Gebiete 55 (237-254).

[2] Blumenthal, R. M. and Getoor, R. K. (1968). Markov Processes and Potential Theory. Academic Press.

[3] Caffarelli, L. A. (1977). The regularity of free boundaries in higher dimensions. Acta Math. 139 (155-184).

[4] Caffarelli, L. A. and Salsa, S. (2005). A Geometric Approach to Free Boundary Problems. Graduate Studies in Mathematics 68, American Mathematical Society.

[5] Cox, A. M. G. and Peskir, G. (2015). Embedding laws in diffusions by functions of time. Ann. Probab. 43 (2481-2510).

[6] Christensen, S. and Salminen, P. (2018). Multidimensional investment problem. Math. Financ. Econ. 12 (75-95).

[7] De Angelis, T. (2015). A note on the continuity of free-boundaries in finite-horizon optimal stopping problems for one-dimensional diffusions. SIAM J. Control Optim. 53 $(167-184)$.

[8] De Angelis, T. Federico, S. and Ferrari, G. (2017). Optimal boundary surface for irreversible investment with stochastic costs. Math. Oper. Res. 42 (1135-1161). 
[9] De Angelis, T. and Peskir, G. (2016). Global $C^{1}$ regularity of the value function in optimal stopping problems. Research Report No. 13, Probab. Statist. Group Manchester (to appear).

[10] De Angelis, T. and Stabile, G. (2017). On Lipschitz continuous optimal stopping boundaries. Preprint arXiv: 1701.07491.

[11] Du Toit, J. and Peskir, G. (2009). Selling a stock at the ultimate maximum. Ann. Appl. Probab. 19, (983-1014).

[12] Ferrari, G. (2017). On the optimal management of public debt: A singular stochastic control problem. Preprint arXiv:1607.04153. To appear in SIAM J. Control Optim.

[13] Friedman, A. (1964). Partial Differential Equations of Parabolic Type. Prentice-Hall.

[14] Friedman, A. (1975). Parabolic variational inequalities in one space dimension and smoothness of the free boundary. J. Funct. Anal. 18 (151-176).

[15] Gilbarg, D. and Trudinger, N. S. (2001). Elliptic Partial Differential Equations of the Second Order. Springer.

[16] Johnson, P. and Peskir, G. (2017). Quickest detection problems for Bessel processes. Ann. Appl. Probab. 27 (1003-1056).

[17] Johnson, P. and Peskir, G. (2018). Sequential testing problems for Bessel processes. Trans. Amer. Math. Soc. 370 (2085-2113).

[18] Novikov, A. A. (1971). On moment inequalities for stochastic integrals. Theory Probab. Appl. 16 (538-541).

[19] Peskir, G. (2005). On the American option problem. Math. Finance 15 (169-181).

[20] Peskir, G. (2005). A change-of-variable formula with local time on curves. J. Theoret. Probab. 18 (499-535).

[21] Peskir, G. (2007). A change-of-variable formula with local time on surfaces. Sém. de Probab. XL, Lecture Notes in Math. 1899, Springer (69-96).

[22] Peskir, G. and Shiryaev, A. N. (2006). Optimal Stopping and Free-Boundary Problems. Lectures in Mathematics, ETH Zürich, Birkhäuser.

[23] Revuz, D. and Yor, M. (1999). Continuous Martingales and Brownian Motion. Springer.

[24] Shiryaev, A. N. (1978). Optimal Stopping Rules. Springer.

Goran Peskir

School of Mathematics

The University of Manchester

Oxford Road

Manchester M13 9PL

United Kingdom

goran@maths.man.ac.uk 